\title{
Molecular Dynamics in Formation Process of Single-Walled Carbon Nanotubes
}

\author{
Yasushi SHIBUTA and Shigeo MARUYAMA
}

Department of Mechanical Engineering, The University of Tokyo, Japan

\begin{abstract}
The mechanism in the nucleation and formation of single-walled carbon nanotubes (SWNTs) was investigated using molecular dynamics simulations. When initial state was chosen so that carbon and nickel atoms were randomly distributed in a simulation domain, the formation of random cage structure made up of carbon atoms, which had a few nickel atoms inside it, was observed at the time of 6 ns. The nickel atoms, which move both inside or on the surface of the cage, were seen to be preventing the complete closure of the cage and its anneal into the fullerene structure. Further, in order to observe longer time-scale growth process, the simulation cell was artificially shrunk with a progress of simulation so that collisions between precursor clusters were promoted to comply with the limitation in the calculation time. Collisions of the imperfect random-cage clusters lead to an elongated tubular cage structure, which could be regarded as an initiation of SWNTs. The simulation results were compared with FT-ICR mass spectra of the positive clusters generated by laser-vaporization supersonic-expansion cluster beam source.
\end{abstract}


Key words: Molecular Dynamics Simulation, Growth Mechanism, SWNTs, Brenner Potential, Nickel Atoms

\section{Introduction}

... 1st order head

The discoveries of multi-walled carbon nanotubes (MWNTs) [1] in 1991 and single-walled carbon nanotubes (SWNTs) [2] in 1993 have opened new research area with these novel carbon materials. In the earliest period, the synthesis of SWNTs was carried out with pulsed laser-oven technique using Ni/Co doped graphite rod [3] or electric arc-discharge technique using $\mathrm{Ni} / \mathrm{Y}$ doped graphite rod [4]. In the last few years, the catalytic chemical vapor deposition (CCVD) technique has been extensively investigated [5] due to its potential toward large-scale production of SWNTs. Several remarkable mechanical/electrical properties of SWNT have been revealed so far, such as a metal-semiconductor duality determined by its chiral number $(n, m)$ that is uniquely assigned to each chiral structure of SWNTs [6].

It is crucially important to elucidate the formation mechanism of SWNTs for the production of larger amount of SWNTs with higher quality and purity (and, if possible, controlled chirality), as well as for the theoretical interest on self-assembly formation of such perfect, elongated structure made up of carbon. Investigations on this formation mechanism have been intensively studied through laser-furnace and arc-discharge experiments, and several findings were obtained from them: For example, the diameter distribution of SWNTs shifts toward smaller side when $\mathrm{Rh} / \mathrm{Pd}$ was used instead of $\mathrm{Ni} / \mathrm{Co}$ for catalyst [7]. It is also found that the higher temperature during the reaction leads to the larger diameter distribution in produced SWNTs [8]. Further, the laser 
plume emission and light scattering were observed during the laser ablation experiment [9-11] to examine the detailed growth process of SWNTs. Based on these experiments, various models for SWNTs formation have been proposed.

In earlier time, Smalley et al. [3] proposed the "Scooter model” for growth mechanism of SWNTs synthesized with laser ablation technique. In this model, one or more metal atoms were assumed to sit on the open edge of a precursor fullerene cluster, which was thought to contribute to the uniform diameter of the tubes, and the role of catalytic metal was thought to prevent a formation of ‘carbon pentagon' by scooting around the open edge. Later, they admitted that the scooter model was applicable only to the order of precursor carbon clusters, and that in the steady growth stage the carbon atoms were supplied from metal clusters that have similar magnitude of SWNT diameters. On the other hand, Yudasaka et al. [12] tested several metal species and their alloys, with detailed examination on their phase diagrams, to elucidate the effect of catalyst choice on SWNT synthesis using laser-furnace technique. From these experiments, they suggested "Metal-particle model", in which the droplets of carbon-containing catalytic metal were formed during the laser-ablation and in the subsequent cooling stage the carbon atoms were separated from the metal nuclei and emerged to form SWNTs. Further, Kataura et al [7] proposed the "fullerene-cap model”, where they speculated that carbon clusters or fullerenes might be eaten by metal particles serving as nuclei for SWNT growth, based on the reason that the growth conditions of fullerene and SWNT were similar and that the diameter distribution of SWNTs correlates with that of fullerenes.

In contrast, theoretical study on the generation mechanism of SWNTs is not straightforward because the size and time scale of actual experimental system is too large to reproduce in the current computational capacities. In some of previous classical 
molecular dynamics calculations [13, 14], a limited portion of generation mechanism was examined using Brenner potential [15], however, there have been almost no report including the role of catalytic metal. This is because there has been no reliable classical potential function between metal and carbon atoms. We constructed a classical potential function between carbon clusters and several metal atoms (La, Sc and Ni) based on DFT calculations of small metal-carbon binary clusters for the simulation of the formation process of the endohedral metallofullerene $[16,17]$. In this paper, using these potential functions, molecular dynamics simulations were performed with carbon and nickel system for the precursor clusters of SWNTs. In addition, by compressing the calculation cell to enhance the collision of those precursor clusters, the possible structure as the result of successive collisions was explored. Further, the comparative experiments were employed by Fourier Transform Ion Cyclotron Resonance (FT-ICR) mass spectrometer $[18,19]$ to study the metal-carbon binary clusters generated by the laser-vaporization of metal-doped carbon material used for the laser-furnace production of SWNTs.

\section{Nomenclature}

\footnotetext{
$B_{i j}^{*}$ : valence function(for carbon - carbon)

$B_{i}^{*}: \quad$ valence function(for carbon - metal)

$b: \quad$ potential parameter

$D_{e}: \quad$ depth of potential

$E_{b}$ : binding energy

$f: \quad$ cut-off function

$N^{\mathrm{C}} \quad$ coordination number of carbon atoms

$N^{\mathrm{M}}{ }_{i} \quad$ coordination number of metal atoms
} 
$R_{e} \quad$ interatomic distance of equilibrium

$r_{i j} \quad$ distance between atom $i$ and $j$

$S$ : $\quad$ potential parameter

$T_{\mathrm{c}}$ : control temperature

$t: \quad$ time

$V_{\mathrm{A}} \quad$ attractive term

$V_{\mathrm{R}} \quad$ repulsive term

$\beta$ : $\quad$ potential parameter

$\delta$ : potential parameter

\section{Simulation Technique}

We employed the Brenner potential [15] in its simplified form [20-23] for carbon-carbon covalent bonding. This potential could describe variety of small hydrocarbons and graphite and diamond lattices. The total potential energy of the system $E_{\mathrm{b}}$ was expressed as the sum of the bonding energy of each bond between carbon atoms $i$ and $j$.

$$
E_{\mathrm{b}}=\sum_{i} \sum_{j(i<j)}\left[V_{\mathrm{R}}\left(r_{i j}\right)-B_{i j}^{*} V_{\mathrm{A}}\left(r_{i j}\right)\right]
$$

where $V_{\mathrm{R}}(r)$ and $V_{\mathrm{A}}(r)$ were repulsive and attractive force term, respectively. The Morse type form with a cut-off function $f(r)$ expressed these terms.

$$
\begin{aligned}
& V_{\mathrm{R}}=f(r) \frac{D_{\mathrm{e}}}{S-1} \exp \left\{-\beta \sqrt{2 S}\left(r-R_{\mathrm{e}}\right)\right\} \\
& V_{\mathrm{A}}=f(r) \frac{D_{\mathrm{e}} S}{S-1} \exp \left\{-\beta \sqrt{2 / S}\left(r-R_{\mathrm{e}}\right)\right\} \\
& f(r)= \begin{cases}1 & \left(r<R_{1}\right) \\
\frac{1}{2}\left(1+\cos \frac{r-R_{1}}{R_{2}-R_{1}} \pi\right) & \left(R_{1}<r<R_{2}\right) \\
0 & \left(r>R_{2}\right)\end{cases}
\end{aligned}
$$


The effect of the bonding condition of each atoms was taken into account through $B^{*}{ }_{i j}$ term which was the function of angle $\theta_{i j k}$ between bonds $i-j$ and $i-k$.

$$
B_{i j}^{*}=\frac{B_{i j}+B_{j i}}{2}, \quad B_{i j}=\left(1+\sum_{k \neq \neq i, j)} G_{c}\left(\theta_{i j k}\right) f\left(r_{i k}\right)\right)^{-\delta},
$$

where

$$
G_{c}(\theta)=a_{0}\left(1+\frac{c_{0}^{2}}{a_{0}^{2}}-\frac{c_{0}^{2}}{d_{0}^{2}+(1+\cos \theta)^{2}}\right)
$$

We had constructed the classical potential function between carbon clusters and several metal atoms (La, Sc and Ni) based on DFT calculation of small metal-cabon binary clusters for the simulation of the formation process of the endohedral metallofullerene $[16,17]$. Here, we employed this potential for the Ni-carbon and Ni-Ni interaction.

Ni-carbon multi-body potential were constructed as functions of the carbon coordinate number of a metal atom. The carbon coordination number of a metal atom $N^{\mathrm{C}}$ is defined using the cutoff function $f(r)$, and the additional term $B^{*}$ was expressed as the function of the coordinate number. Here, the effect of the angle among bonds is ignored.

$$
\begin{aligned}
& N^{C}=1+\sum_{\operatorname{carbon} k(\neq j)} f\left(r_{i k}\right) \\
& B^{*}=\left\{1+b\left(N^{\mathrm{C}}-1\right)^{\delta}\right\}
\end{aligned}
$$

For metal-metal interactions, we expressed the equilibrium binding energy $D_{\mathrm{e}}$ and the bond length $R_{\mathrm{e}}$ as direct function of the metal coordinate number $N_{i j}$, instead of using the additional term $B^{*}$.

$$
\begin{aligned}
& N_{i}^{\mathrm{M}}=1+\sum_{\text {metal } k(\neq j)} f\left(r_{i k}\right), \quad N_{i j}=\frac{N_{i}^{\mathrm{M}}+N_{j}^{\mathrm{M}}}{2} \\
& D_{\mathrm{e}}\left(N_{i j}\right)=D_{\mathrm{e} 1}+D_{\mathrm{e} 2} \exp \left\{-C_{\mathrm{D}}\left(N_{i j}-1\right)\right\} \\
& R_{\mathrm{e}}\left(N_{i j}\right)=R_{\mathrm{e} 1}-R_{\mathrm{e} 2} \exp \left\{-C_{\mathrm{R}}\left(N_{i j}-1\right)\right\}
\end{aligned}
$$

Constants are summarized in Table 1 and 2. 
The high density of the system was compensated with the very rapid cooling with the temperature control. Through this rapid temperature control, energy distribution between translation, rotation, and vibration may be unrealistically in non-equilibrium. Hence, the special temperature control method [20-23] that control translational, rotational and vibrational temperature of the system independently was employed so that the difference between the temperature of the system and Tc was reduced by $60 \%$ in 1 ps. Velocity Verlet method was employed to integrate the classical equation of motion with the time step of 0.5 fs.

[ Table 1 ]

[ Table 2 ]
... instruction for the position of figures and tables

... instruction for the position of figures and tables
3. Molecular Dynamics Simulation

\subsection{Initial clustering stage}

... 1st order head

... 2nd order head

As for the initial condition, the completely random vapor mixture of 2500 carbon atoms and 25 nickel atoms was allocated in $585 \AA$ cubic fully-periodic simulation cell. The control temperature was set to $3000 \mathrm{~K}$. Figure 1 shows a snapshots after 6 ns molecular dynamics calculation. Many relatively large clusters up to about 200 carbon atoms and a few metal atoms were observed. Carbon clusters tended to be spherical random cage structure with a few metal atoms at around the defect vacancy, which prevented the cage structure from the complete closure.

[ Figure 1] ... instruction for the position of figures and tables

As in our empty fullerene [20-23] and metallofullerene studies [16,17], cluster structures in Figure 1 were not well annealed. An example of the annealing simulation of the Ni-attached carbon cluster is shown in Figure 2. As clearly seen in Figure 2, quasi-periodic motion of the $\mathrm{Ni}$ atom flipping inside and outside of the carbon cage at 
the interval of 1 to 10 ns were observed. The possibility of this meta-stable structure should be confirmed with more accurate $a b$ initio level calculations. The existence of the $\mathrm{Ni}$ atom prevented the cage from closing to fullerene. In case that $\mathrm{Ni}$ atom was eliminated in this annealing process, the cage was annealed to $\mathrm{C}_{60}$ structure.

\section{[ Figure 2 ] ... instruction for the position of figures and tables}

\subsection{Collisions of cage carbon clusters leading to the tubular structure}

In the real generation simulation of SWNTs, it is expected that those clusters experience later collisions at lower temperature and lower frequency as they expand. However, because of the computational limitations, we had to slowly shrink the simulation cell to enhance the collisions. The shrinking rate in table 3 was much slower than the typical translational velocity of clusters. The large particle with some tubular structure was obtained after collisions of clusters. With the condition 1, the large particle made of almost all carbon atoms was obtained after 6.9 ns. Sequential annealing at $2500 \mathrm{~K}$ changed the collided cluster into bulge structure as in Figure 3 and Figure 4 (a). Furthermore, the bulge structure changed the cone structure after sequential annealing at $2500 \mathrm{~K}$ (Figure 4 (b)), in contrast the bulge structure retained in annealing at $1500 \mathrm{~K}$ (Figure 4 (c)) that did not grow to tubular structure after additional $10 \mathrm{~ns}$ annealing. Hence it is not reasonable to nucleate from the bulge to the tube.

\section{[ Figure 3 ] ... instruction for the position of figures and tables}

\section{[ Figure 4 ] ... instruction for the position of figures and tables}

With the condition 2, the longer tubular structure was obtained as in Figure 3 (b). Additional annealing about $30 \mathrm{~ns}$ at $2500 \mathrm{~K}$ urged the bond switching to straight tube structure as in Figure 5. The structure of the particle depends on the temperature, as only temperature condition differs between condition 1 and 2 .

\section{[ Figure 5]


Figure 6 shows the growth process of the typical tubular cluster from shrinking simulation with the condition 3, the shrinking rate of which is tenth. Even though the structure shown in Figure 6 is rather ugly, one can see that the tubular structure has grown longer by the collision and the coalescence. Ni atoms were slowly assembling to form Ni clusters, and they were diffusing around until finding the most stable position at hemi-half-fullerene cap area.

\section{[ Figure 6 ] ... instruction for the position of figures and tables}

Part of the annealing tendency was examined by separate simulations as shown in Figure 7. An intermediate structure appeared in Figure 6 (8 ns) was picked up for this annealing. Comparison of annealing effect with different temperatures (2000 K and 2500K) for 50 ns is shown in Figure 7. The tubular structure became thick at the narrow part and most straight due to annealing. And, the clustering of $\mathrm{Ni}$ atoms was observed for the $2500 \mathrm{~K}$ case. The metal cluster tended to come to the curved carbon area. Unfortunately, in case of Figure 7, the metal cluster was trapped in curved defect part of the tubular structure. Since the narrow part of the tubular structure was usually Ni free, the tubular structure can be annealed to SWNT structure.

[ Figure 7 ] … instruction for the position of figures and tables

\section{Comparative experiments by FT-ICR mass spectrometer $\quad .$. 1st order head}

In order to compare foregoing simulation results with the experimental data, the metal-carbon binary clusters generated from the metal-doped graphite, which are the typical materials used in laser-furnace or arc-discharge technique, were studied by FT-ICR $[18,19]$ experiments. Figure 8 presents sample spectra of detected positively charged cluster ions. In this figure, the spectra obtained from several laser-vaporized clusters were compared for the cases of (a) pure, (b) Ni/Co (0.6 at. \% each) doped, (c) 
Rh/Pd (1.0 at. \% each) doped, and (d) Ni (1.2 at. \%) doped graphite sources. As for the pure graphite, even-numbered clusters prevail over the odd ones in the range of $\mathrm{C}_{34}$ to $\mathrm{C}_{50}$, while the larger odd ones were barely detected. This is due to the restriction arising from the Euler's theorem of geometry, which requires the even-numbered cluster to build a closed cage consists only of the three-handed carbon atoms (i.e. $s p^{2}$ hybrid orbital). On the other hand, metal-doped graphite samples were much easier to generate small carbon clusters than pure graphite in the range of $\mathrm{C}_{20}$ to $\mathrm{C}_{45}$ and metal-doped clusters were also observed. Furthermore, since the odd-numbered clusters were observed in the range, it is concluded that metal catalyst prevents the cluster from the complete closure and dangling bonds retains reactivity for continuous collisions for the tubular structure. Our results from molecular dynamics calculation were in good agreement with this tendency.

[ Figure 8 ] ... instruction for the position of figures and tables

\section{Conclusions}

... 1st order head

From molecular dynamics simulations and comparative experiments by FT-ICR mass spectrometer, the growth mechanism of SWNTs in laser-furnace and arc discharge technique is suggested as follows. At the clustering stage, metal atoms tend to stay at around the defect vacancy of carbon cage structure, and therefore prevent the cage from closing to fullerene. Then, the random cage carbon clusters can make further growth by colliding with each other at the large defect area of the cage. The collision leads to the elongated tubular structure. Given the enough time for diffusion of metal atoms and network annealing, we expect that the structure becomes a straight SWNT with metal clusters at each end. 
The author would like to thank Dr. Yasutaka Yamaguchi (Osaka Univ.) for their help in molecular dynamics simulations; Dr. Masamichi Kohno (AIST), Mr. Shuhei Inoue (The Univ. of Tokyo) and Fumihiro Hirokawa (The Univ. of Tokyo) for comparative experiments by FT-ICR mass spectrometer. Part of this work was supported by Grant-in-Aid for Scientific Research (B)(No. 13555050) from the Ministry of Education, Culture, Sports, Science and Technology, Japan.

\section{Literature Cited}

1. Iijima, S., “Herical Microtubules of Graphitic Carbon”, Nature, 1991;354:56-58.

2. Iijima, S. and Ichihashi, "Single-Shell Carbon Nanotubes of 1-nm Diameter", Nature, 1993;363:603-605.

3. Thess, A., Lee, R., Nikolaev, P., Dai, H., Petit, P., Robert, J., Xu, C., Lee, Y. H., Kim, S. G., Rinzler, A. G., Colbert, D. T., Scuseria, G. E., Tománek, D., Fischer, J. E., Smalley, R. E., "Crystalline Ropes of Metallic Carbon Nanotubes”, Science, 1996;273:483-487.

4. Journet, C., Maser, W. K., Bernier, P., Loiseau, A., de la Chapelle, M. L., Lefrant, S., Deniard, P., Lee, R., Fisher, J. E., “Large-Scale Production of Single-Walled Carbon Nanotubes by the Electric-Arc Technique”, Nature, 1997;388:756-758.

5. Maruyama. S., "Generation Technique and Formation Mechanism of Single Walled Carbon Nanotubes”, Trans JSRC, 2002;73:22-27. 
6. Saito. R., Dresselhaus, G., Dresselhaus, M. S., "Physical Properties of Carbon Nanotubes”, Imperial College Press, 1998, 37.

7. Katarura, H., Kumazawa, Y., Maniwa, Y., Ohtsuka, Y., Sen, R., Suzuki, S., Achiba, Y., "Diameter Control of Single-Walled Carbon Nanotubes”, Carbon, 2000;38:1691-1697.

8. Bandow, S., Asaka, S., Saito, Y., Rao, A. M., Grigorian, L., Richter, E., Eklund, P. C., "Effect of the Growth Temperature on the Diameter Distribution and Chirality of Single-Wall Carbon Nanotubes”, Phys. Rev. Lett.,1998;80:3779-3782.

9. Kokai, F., Takahashi, K., Yudasaka, M., Iijima, S., "Lasaer Ablation of Graphite-Co/Ni and Growth of Single-Wall Carbon Nanotubes in Vortexes Formed in an Ar Atmosphere”, J. Phys. Chem. B, 2000;104, 6777-6784.

10. Sen., R., Ohtsuka, Y., Ishigaki, T., Kasuya, D., Suzuki, S., Kataura, H., Achiba, Y., "Time Period for the Growth of Single-Wall Carbon Nanotubes in the Laser Ablation Process: Evidence from Gas Dynamics Studies and Time Resolved Imaging”, Chem. Phys. Lett., 2000;332:467-473.

11. Scott, C. D., Arepalli, S., Nikolaev, P., Smalley, R. E., "Growth Mechanisms for Single-Wall Carbon Nanotubes in a Laser-Ablation Process”, Appl. Phys. A, 2001:72:573-580.

12. Yudasaka, M., Yamada, R., Sensui, N., Wilkins, T., Ichihashi, T., Iijima, S., "Mechanism of the Effect of NiCo, Ni, and Co Catalysts of the Yield of Single-Wall Carbon Nanotubes Formed by Plused Nd:YAG Laser Ablation”, J. Phys. Chem. B, 1999;103:6224-6229.

13. Maiti, A., Brabec, C. J., Bernholc, J., “Theory of Carbon Nanotube Growth”, Phys. Rev. B, 1995;52:14850-14858. 
14. Maiti, A., Brabec, C. J., Bernholc, J., "Kinetics of Metal-Catalyzed Growth of Single-Walled Carbon Nanotube Growth” Phys. Rev. B, 1997;55:6097-6100.

15. Brenner, D. W., "Empirical Potential for Hydrocarbons for Use in Simulating the Chemical Vapor Deposition if Diamond Films”, Phys. Rev. B., 1990;42:9458-9471.

16. Yamaguchi, Y., Hori, S., Maruyama, S., "Molecular Dynamics Simulation of Formation of Metal-Containing Fullerene”, Trans JSME Ser B, 1999;65-630:421-436.

17. Yamaguchi, Y., Maruyama. S., “A Molecular Dynamics Study on the Formation of Metallofullerene”, Euro. Phys. J. D, 1999;9:385-388.

18. Maruyama, S., Anderson, L. R., Smalley, R. E., “Direct Injection Supersonic Cluster Beam Source for FT-ICR Studied of Clusters”, Rev. Sci. Instrum., 1990;61:3686-3693.

19. Maruyama, S., "Perspectives of Fullerene Nanotechnology", Kluwer Academic Publishers, 2002:131-142.

20. Yamaguchi. Y, Maruyama. S, "Molecular Dynamics of the Formation Process of Fullerene (1 Report, The Formation of a Caged Structure and Controlled Temperature)”, Trans JSME Ser B, 1997;63-611:2398-2404.

21. Yamaguchi. Y, Maruyama. S, “A Molecular Dynamics Simulation of the Fullerene Formation Process”, Chem. Phys. Lett., 1998;286:334-342.

22. Maruyama, S., Yamaguchi. Y, “A Molecular Dynamics of the Formation Process of Fullerene (2nd Report, Annealing to the Perfect $\mathrm{C}_{60}$ Structure”, Trans JSME Ser B, 1997;63-611:2405-2412.

23. Maruyama, S., Yamaguchi. Y, “A Molecular Dynamics Demonstration of Annealing to a Perfect $C_{60}$ Structure”, Chem. Phys. Lett., 1998;286:343-349. 


\section{Figure and Table Captions}

Fig. 1. A snapshot of clustering process at $6 \mathrm{~ns}$.

Fig. 2. Annealing of $\mathrm{NiC}_{60}$.

Fig. 3. Structure of the particles with conditions $1 \& 2$.

Fig. 4. Bulge structure with condition 1.

Fig. 5. Annealing of imperfect tube with the condition 2. (All carbon atoms with three-coordination are not shown.)

Fig. 6. Growth process of a tubular structure by successive collisions of imperfect cage clusters.

Fig. 7. Annealing at $2000 \mathrm{~K}$ and $2500 \mathrm{~K}$ of intermediate structure.

Fig. 8. Mass spectra of positive ions by FT-ICR.

Table 1. Potential parameters for carbon-carbon and Ni-C interactions.

Table 2. Potential parameters for Ni-Ni interactions.

Table 3. Conditions of shrinking process. 


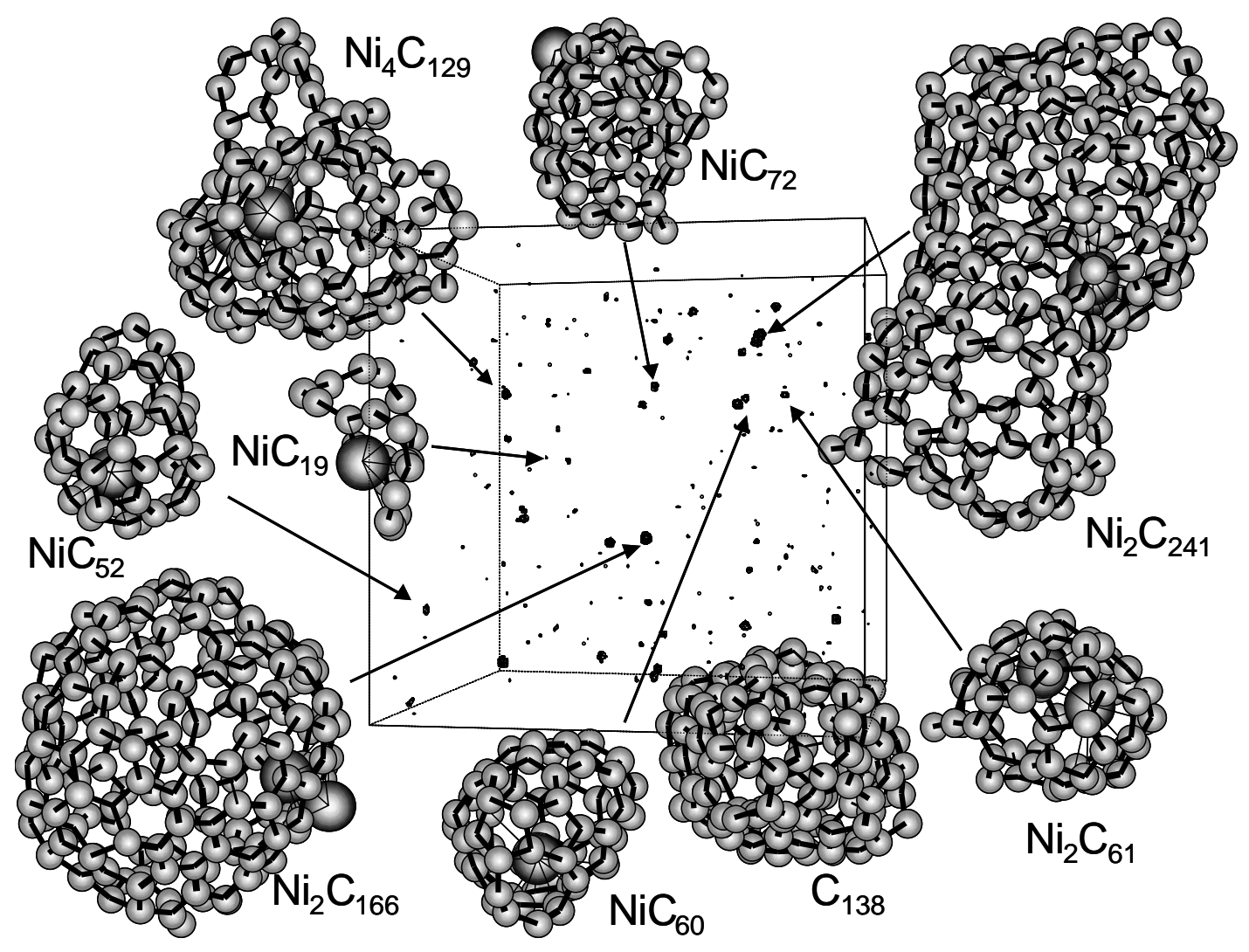

Fig. 1. A Snapshot of clustering process at 6 ns. 


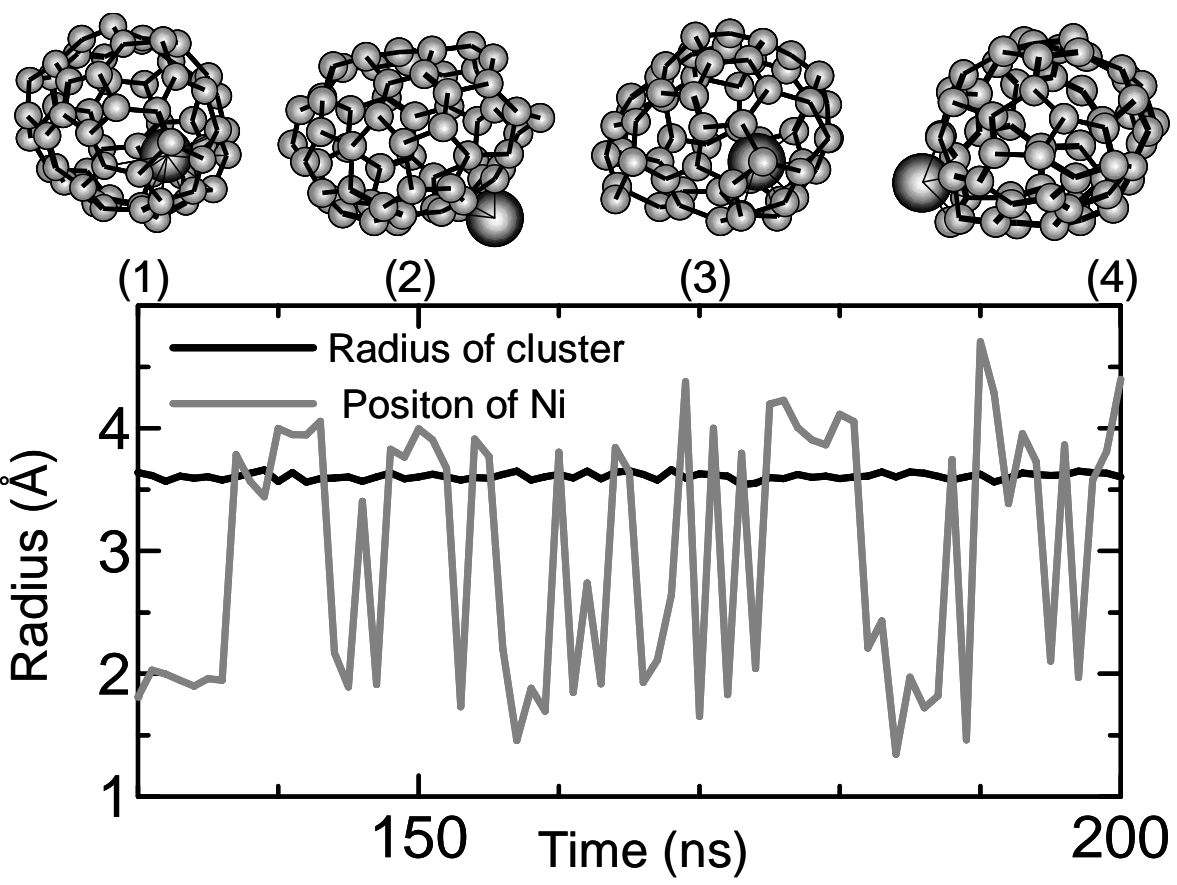

Fig. 2. Annealing of $\mathrm{NiC}_{60}$. 


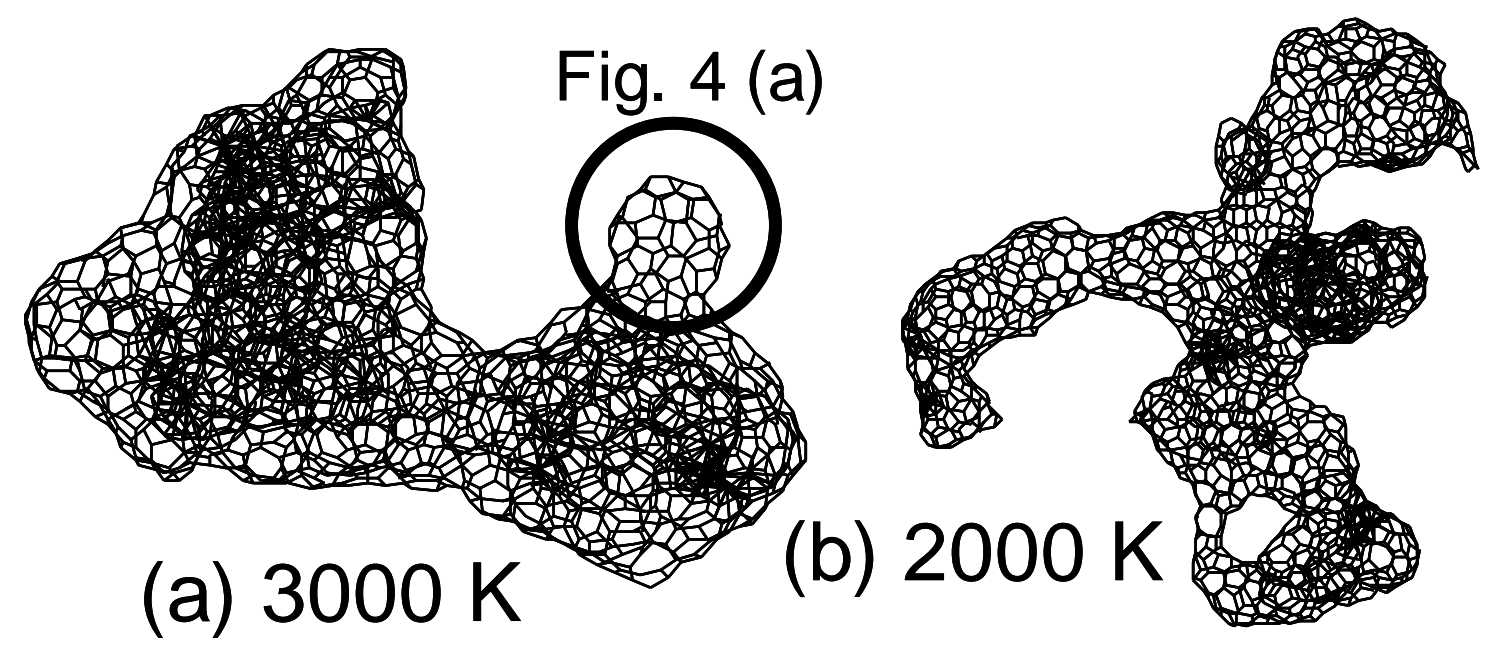

Fig. 3. Structure of the particles with condition $1 \& 2$. 


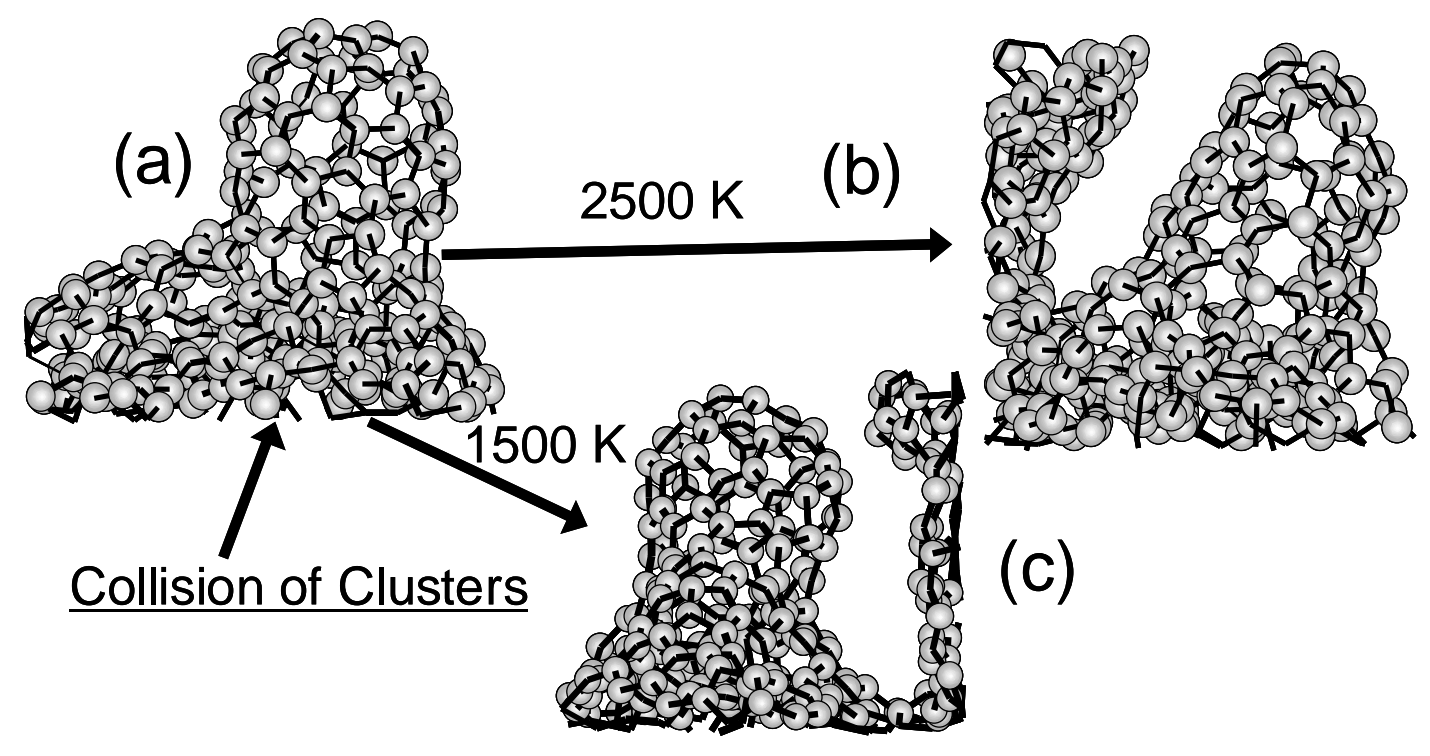

Fig. 4. Bulge structure with condition 1. 


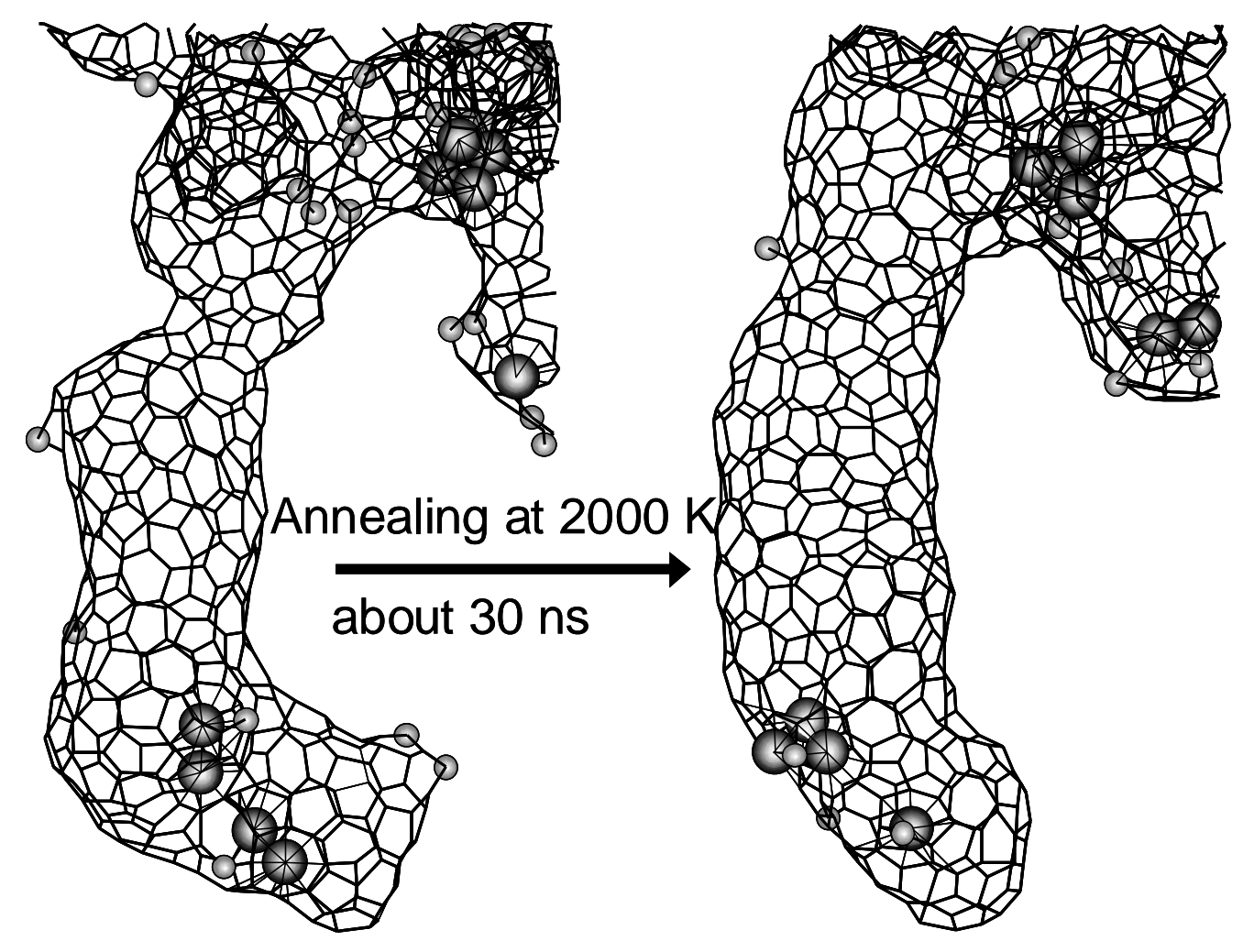

Fig. 5. Annealing of imperfect tube with the condition 2.

(All carbon atoms with three-coordination are not shown.) 


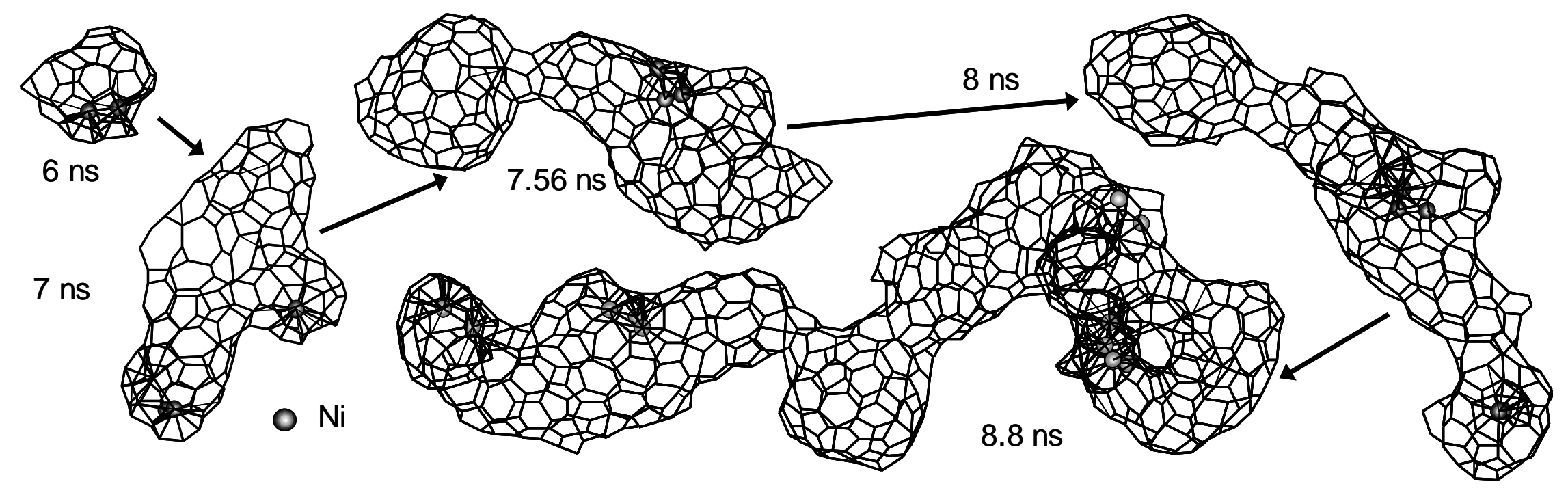

Fig. 6. Growth process of a tubular structure by successive collisions of imperfect cage clusters. 


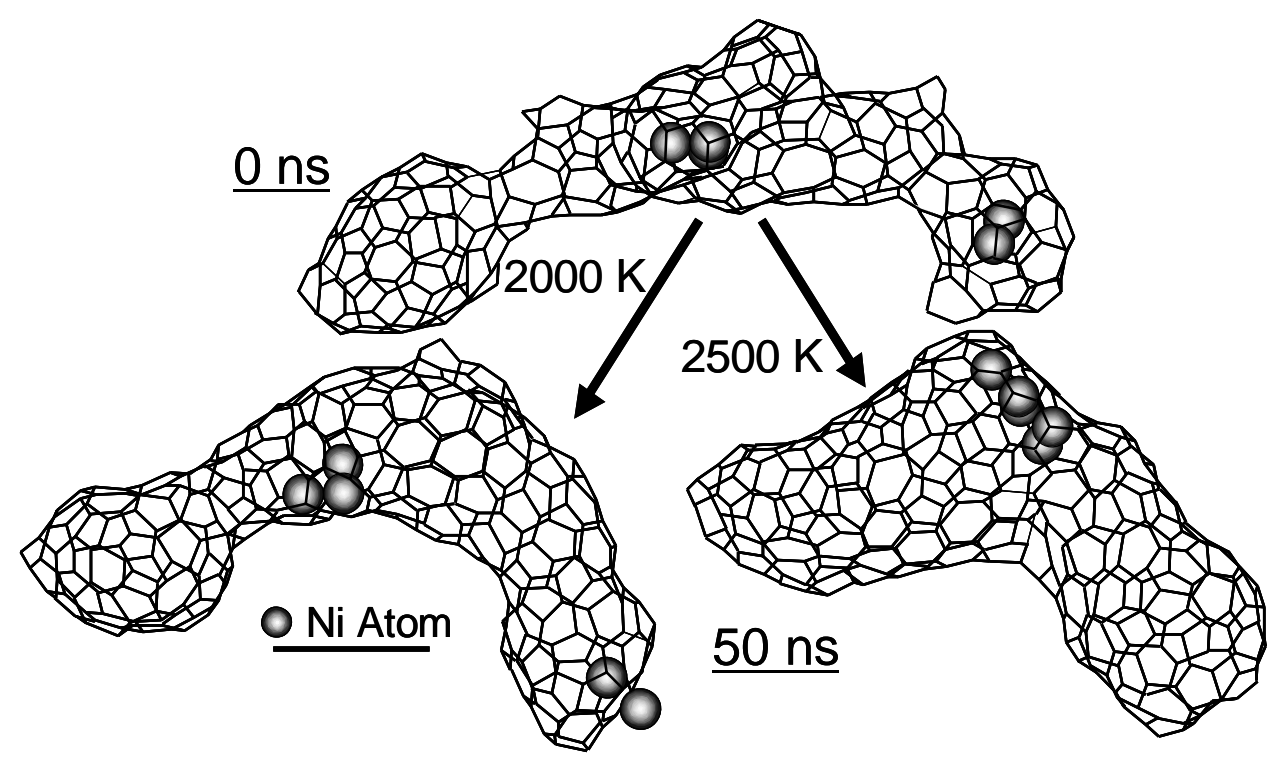

Fig. 7. Annealing at $2000 \mathrm{~K}$ and $2500 \mathrm{~K}$ of intermediate structure. 


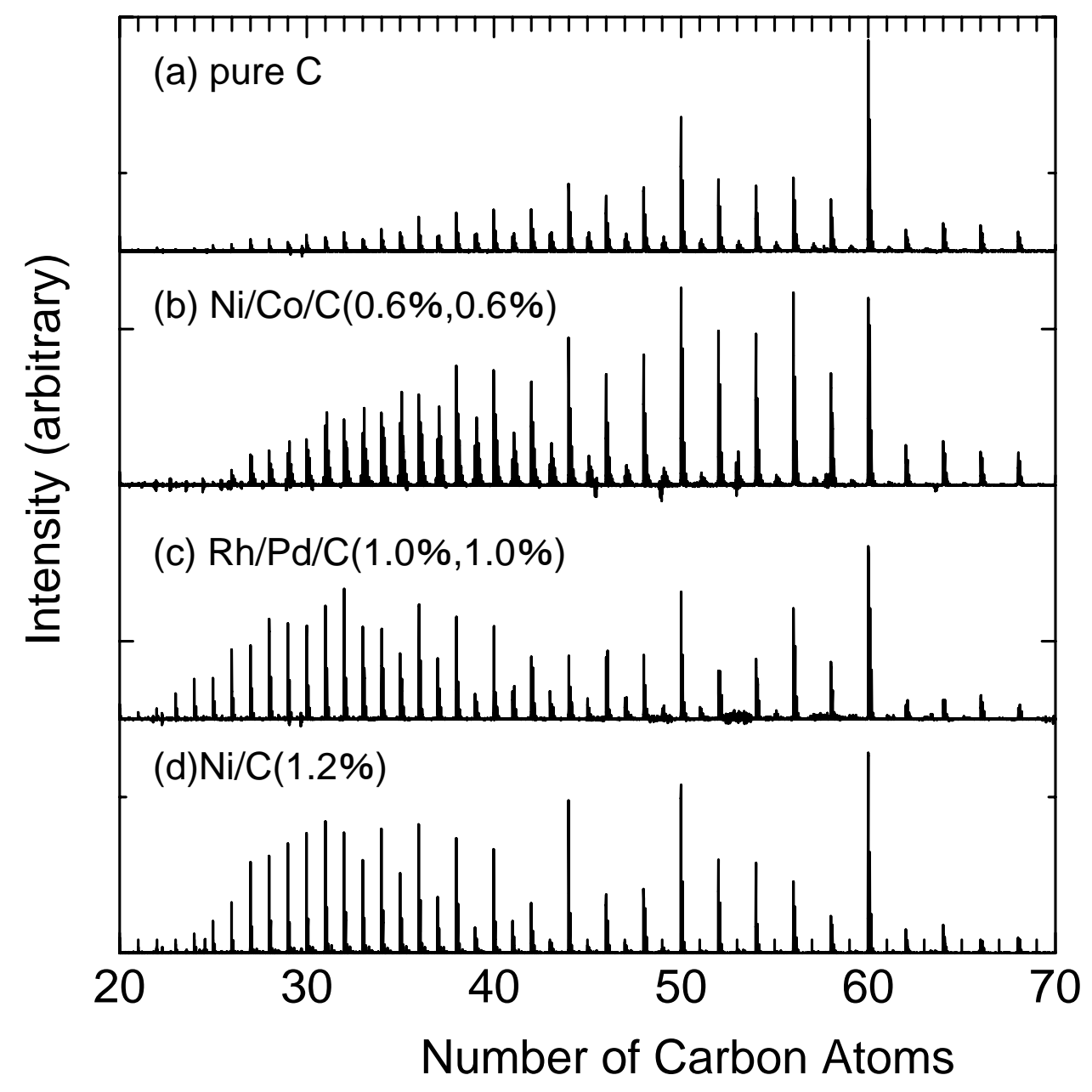

Fig. 8. Mass spectra of positive ions by FT-ICR. 
Table 1. Potential parameters for carbon-carbon and Ni-C interactions.

\begin{tabular}{|c|c|c|c|c|c|c|c|c|c|c|c|}
\hline & $D_{\mathrm{e}}(\mathrm{eV})$ & $S$ & $\beta(1 / \AA)$ & $R_{\mathrm{e}}(\AA)$ & $R_{1}(\AA)$ & $R_{\mathrm{s}}(\AA)$ & $\delta$ & $a_{0}$ & $c_{0}$ & $d_{0}$ & $b$ \\
\hline C - C & 6.325 & 1.29 & 1.5 & 1.315 & 1.7 & 2.0 & 0.80469 & 0.011304 & 19 & 2.5 & - \\
\hline $\mathrm{Ni}-\mathrm{C}$ & 3.02 & 1.3 & 1.8 & 1.7 & 2.7 & 3.0 & -0.8 & - & - & - & 0.0330 \\
\hline
\end{tabular}

Table 2. Potential Parameters for Ni-Ni interactions.

\begin{tabular}{|c|c|c|c|c|c|c|c|c|c|c|}
\hline & $\mathrm{S}$ & $\beta(1 / \AA)$ & $D_{\mathrm{e} 1}(\mathrm{eV})$ & $D_{\mathrm{e} 2}(\mathrm{eV})$ & $C_{\mathrm{D}}$ & $R_{\mathrm{e} 1}(\AA)$ & $R_{\mathrm{e} 2}(\AA)$ & $C_{\mathrm{R}}$ & $R_{1}(\AA)$ & $R_{2}(\AA)$ \\
\hline $\mathrm{Ni}-\mathrm{Ni}$ & 1.3 & 1.55 & 0.74 & 1.423 & 0.365 & 2.520 & 0.304 & 0.200 & 2.7 & 3.2 \\
\hline
\end{tabular}

Table 3. Conditions of shrinking process.

\begin{tabular}{|c|c|c|}
\hline & Max shrink rate (per time step) & Temperature \\
\hline 1 & $6 \times 10^{-4} \AA$ & $3000 \mathrm{~K}$ \\
\hline 2 & $6 \times 10^{-4} \AA$ & $2000 \mathrm{~K}$ \\
\hline 3 & $6 \times 10^{-5} \AA$ & $2000 \mathrm{~K}$ \\
\hline
\end{tabular}

